\title{
Gestión organizacional para la formación doctoral local en Ciencias de la Salud en Holguín
}

\author{
Organization management for the local Ph.D. in Health \\ Sciences studies in Holguín
}

\begin{abstract}
Alcides Abad Ochoa Alonso'; Aida Rodríguez Cabrera ${ }^{\text {II }}$
I Doctor en Ciencias de la Salud. Especialista de II Grado en Administración y Organización de Salud Pública. Universidad de Ciencias Médicas de Holguín. Holguín, Cuba.

${ }^{\mathrm{II} D o c t o r}$ en Ciencias Económicas. Profesor Titular. Escuela Nacional de Salud Pública. La Habana, Cuba.
\end{abstract}

\section{RESUMEN}

Entre las orientaciones del Ministerio de Salud Pública de Cuba está desarrollar estrategias aceleradas para la formación de doctores en ciencias en el sector, fundamentalmente, a nivel local. Por ello, consecuentemente con esta orientación la provincia de Holguín se propuso impulsar el proceso de formación de doctores, en este caso en Ciencias de la Salud, para elevar el nivel de excelencia académica de la Universidad de Ciencias Médicas, como parte de su inserción en el campo internacional y además, como respuesta a la solicitud de los profesionales de la provincia que deseaban obtener este grado científico. Se muestra la estrategia elaborada y su diseño sustentada en un proceso formativo flexible y ajustado a las normas vigentes por la Comisión Nacional de Grados Científicos de Cuba para la obtención del grado de doctor en una especialidad determinada, procedimiento conocido y utilizado por otros sectores, pero prácticamente desconocido en el sector de la salud. Este proceso de formación doctoral es un modelo de transformación académica que agiliza la formación de doctores en ciencias de la salud en Holguín y prevé disponer a corto plazo de un grupo importante de profesores científicamente fortalecidos. A un año de ejecución de la estrategia se observan resultados estimulantes y se han trazado las proyecciones para su sostenibilidad.

Palabras clave: Gestión organizacional, formación doctoral, ciencias de la salud. 


\begin{abstract}
One of the instructions of the Ministry of Public Health is the development of strategies for the intensive formation of Ph.D. in health sciences, mainly at local level. To this end, Holguín province intended to give a boost to the process of Ph.D. formation in the health sciences field, in order to increase the level of academic excellence at the provincial medical science university, as part of its insertion in the international arena, and additionally, as a response to the request of the health professionals in that province. The strategy and its design, based on a flexible formation process adapted to the present standards of the National Commission of Scientific Degrees in Cuba for obtaining Ph.D. in a given specialty, was presented. This procedure is known and used in other sectors, but it is practically unknown in the healthcare sector. This process of Ph.D. formation is a model of academic transformation that speeds up the formation Doctors of health sciences in Holguin, and anticipates short-term availability of a significant group of scientifically sounded professors. After one year of the implementation of this strategy, stimulating results were observed and new projections towards its sustainability have been set.
\end{abstract}

Key words: Organization management, Ph.D. formation, Health sciences.

\title{
INTRODUCCIÓN
}

La Ley Número 1281 de 2 de diciembre de 1974, que establece el Sistema Nacional de Grados Científicos y crea la Comisión Nacional de Grados Científicos (CNGC) desde su inicio se fundamenta, en que en el país existen "condiciones que permiten abordar etapas superiores para la formación de especialistas de alto nivel, a través de la educación de posgrado, mediante un sistema de grados científicos para los graduados universitarios". ${ }^{1}$ Hoy, trascurridos 34 años el sector de la salud no ha estado a la altura de ese planteamiento y la formación de doctores se ha quedado un tanto retrasada con respecto a otros sectores en el país, por ello el Ministerio de Salud Pública (MINSAP) elaboró una estrategia para el desarrollo de los grados científicos, donde se establece la política general que debe seguirse para avanzar en la formación de doctores en el sector y se esbozan las actividades que deben ejecutarse a nivel provincial y municipal.

Las resoluciones y acuerdos emanados de los plenos de la CNGC permiten operacionalizar la conducción de estos procesos. En consonancia con ello el MINSAP, a través del área de posgrado del Viceministerio de Docencia e Investigaciones, a partir de las Normas y Resoluciones vigentes para el desarrollo de los Grados Científicos en la República de Cuba² elaboró en el año 2007 el Plan de Acción Estratégico para el Desarrollo de los Grados Científicos del MINSAP. ${ }^{3}$

Al constituirse la Escuela Nacional de Salud Pública (ENSAP) como Institución Autorizada para el otorgamiento de Grados Científicos en Ciencias de la Salud, resultó pertinente y oportuno diseñar y ejecutar una estrategia para acelerar la obtención del grado científico en esta especialidad, este proceso comenzó a desarrollarse con los profesionales de la salud que cumplen misión internacionalista 
en la República Bolivariana de Venezuela, experiencia que constituye una buena razón para extenderla a otras regiones del país, con las adecuaciones lógicas de ejecutarse en una provincia de Cuba.

A partir de lo anterior se valoró la existencia en la provincia de Holguín de los elementos básicos para el desarrollo de una estrategia de formación doctoral: problemas de investigación, sujetos para llevar a cabo el doctorado (aspirantes y tutores) y un sistema de gestión.

Tal como estableció el MINSAP el desarrollo de la estrategia en los territorios se sustenta en la actuación de los Centros de Educación Médica Superior (CEMS), direcciones provinciales e instituciones de salud a través de las siguientes acciones estratégicas:

1. Establecer una estructura de coordinación para la formación de doctores en cada municipio.

2. Confeccionar plan de formación de doctores de cada institución de salud.

3. Aprovechar las posibilidades que ofrece el banco de problemas de investigación de los territorios de modo que su solución contribuya con la formación de doctores.

4. Creación de soportes de apoyo en función de las necesidades individuales.

Por tanto, en consonancia con la política científica nacional y las proyecciones estratégicas del MINSAP y de la ENSAP, se consideró pertinente desarrollar un proceso de formación doctoral que incluyera modalidades novedosas en el campo de la salud, con la rectoría de la ENSAP, y cuya finalidad declarada consiste en producir profesionales con alto nivel científico y técnico, cultural, salubrista, mentalidad innovadora y creativa, capacidad para resolver problemas de investigación en la provincia y con altos valores éticos. ${ }^{4}$

En el territorio de la provincia de Holguín, se trabaja hoy, con éxitos, por elevar la salud y la calidad de vida de su población. Sin embargo, el desarrollo científico en el campo de la salud pública, al igual que en otras provincias del país, es aún insuficiente, si se mide por el número de doctores en ciencias en esta rama. Los cerca de 15000 profesionales de la salud en el territorio holguinero constituyen el $22 \%$ del total de profesionales de todas las ramas; sin embargo, este sector dispone de solo 12 doctores en ciencias de una especialidad determinada, lo que constituye el 5,4\% del total. ${ }^{5}$ Específicamente en ciencias de la salud solo existe uno.

El objetivo de este artículo es exponer la gestión que se ha llevado a cabo para la formación de 25 doctores en ciencias de la salud en la provincia de Holguín entre los años 2008 y 2012 distribuidos anualmente de la siguiente manera: ocho en el año 2010, siete en el año 2011 y diez en el año 2012; mostrar los resultados obtenidos y las proyecciones existentes.

\section{MÉTODOS}

Para la realización de la estrategia de trabajo se aplicaron las siguientes técnicas, que posibilitaron enriquecer el diagnóstico ya realizado desde el nivel central del MINSAP: ${ }^{6}$ 
- Revisión documental.

- Entrevistas a informantes clave para la determinación de un banco de problemas.

- Técnicas grupales para la elaboración de una matriz DAFO.

\section{RESULTADOS}

\section{En el diseño de la estrategia}

A partir de las técnicas aplicadas se obtuvieron resultados que posibilitaron realizar el siguiente análisis de fuerzas.

\section{Barreras:}

- Falta de información en los profesionales de la salud en la provincia.

- Carencia de un claustro profesoral en el territorio con la presencia de un número importante de doctores en Ciencias de la Salud.

- Ausencia de una gestión sectorial específica hacia el doctorado en Ciencias en general y en particular en Ciencias de la Salud.

- Ubicación geográfica distante del polo con más profesionales con alto grado científico en Ciencias de la Salud.

Oportunidades y fortalezas:

- Decisión de los directivos de la Dirección Provincial de Salud y la Universidad de Ciencias Médicas de Holguín, para implementar el doctorado, asignar un responsable del proyecto para la ejecución y control del proceso, que garantice su ejecución con rigor científico y metodológico, y asegurar los recursos necesarios para su desarrollo exitoso.

- Voluntad en los órganos de dirección estatal de la provincia para apoyar y controlar el desarrollo del doctorado.

- Voluntad de la dirección de la ENSAP y su claustro para colaborar en el desarrollo exitoso del doctorado en la provincia.

- Existencia de doctores en Ciencias Médicas, Filosóficas, Psicológicas y Pedagógicas en el territorio, cuyas características específicas tributan a su valoración como posibles tutores y asesores en el proceso de formación doctoral.

- Interés y compromisos de colaboración por parte de la Delegación Territorial del Ministerio de Ciencia, Tecnología y Medio Ambiente (CITMA), así como de otras entidades de Ciencia e Innovación Tecnológica y los Centros de Educación Superior (CES) de la provincia.

- Existencia de un grupo de profesionales con trayectoria, perfil y firme voluntad para cumplir los requerimientos del proceso de formación doctoral. 
- Existencia de un doctor en Ciencias de la Salud de la provincia con ubicación transitoria en la ENSAP, con posibilidades de asumir como tarea principal la organización y desarrollo de esta misión.

A partir de los elementos descritos se consideró la necesidad y la posibilidad de implementar en el territorio de la provincia de Holguín la Estrategia que el Plan Nacional enuncia como el proceso de formación científica del aspirante, con la colaboración de otros profesionales e instituciones, dirigida hacia los siguientes componentes:

- Formación teórica en Ciencias de la Salud.

- Formación en metodología de la investigación.

- Ejecución de la investigación.

- Preparación y defensa de la tesis.

Además, se establecieron como principios para ejecutar la estrategia de formación doctoral los siguientes:

- Las estrategias y tácticas para la organización del proceso estarán subordinadas a las prioridades ya establecidas al claustro de la ENSAP.

- Cumplimiento riguroso e inviolable de los requerimientos docentes metodológicos en todos los procesos.

- La Dirección Provincial de Salud y la Universidad de Ciencias Médicas de Holguín garantizarán la transportación y alojamiento confortable para los profesores, así como toda la logística requerida.

\section{Estrategia de intervención organizacional}

Entre los componentes principales de esta estrategia se encuentran los siguientes:

- Establecimiento formal de los términos de cooperación entre la Universidad de Ciencias Médicas de Holguín, como institución ejecutora principal, y las instituciones participantes, a saber: ENSAP y Universidad de Holguín "Oscar Lucero Moya" (UHOLM).

- Coordinación de acciones colaborativas específicas con la delegación territorial del CITMA, Unidades de Ciencia y Técnica y otros CES.

- Proceso de selección de aspirantes y tutores.

- Elaboración del plan de desarrollo individual de los aspirantes y del cronograma de trabajo grupal.

- Elaboración, ejecución y control del plan de acción de las actividades metodológicas, docentes y organizacionales del proceso.

- Designación de un Doctor en Ciencias de la Salud de Holguín, con estancia temporal en la ENSAP, como coordinador permanente de la estrategia. 
Para su alcance se implementaron las siguientes acciones:

- Programación de asesorías metodológicas ajustadas a las prioridades de trabajo de la ENSAP.

- Coordinación con los presidentes de los tribunales de Problemas Sociales de las Ciencias y la tecnología (PSCYT) e Idiomas de la UHOLM, para asumir los cursos y ejercicios de mínimos en las respectivas disciplinas a los futuros aspirantes.

- Identificación de doctores en ciencias de determinada especialidad del territorio, para incorporarse como pares a Doctores en Ciencias de la Salud, lo que pudiera permitir la complementación de experiencias y enfoques en asesorías metodológicas y/o tutorías.

- Selección rigurosa de futuros aspirantes (entre 20 y 25).

- Establecimiento de las vías para la participación de los profesionales interesados y con posibilidades para obtener el grado en encuentros de carácter científico pertinentes con los temas de sus investigaciones.

- Instrumentación del apoyo de las instituciones involucradas en el proceso.

- Gestión de la disponibilidad de fondo de tiempo de los profesionales aceptados en el proceso para el desarrollo de la investigación según cronograma y necesidades de cada uno.

- Aseguramiento logístico para la realización de cursos sobre Ciencias de la Salud, metodología de la investigación o en temas complementarios a la tesis, ejecución de exámenes de mínimos, talleres de tesis, consultas con expertos del tema en otras provincias, ejercicios pre-defensas y defensas de tesis.

- Creación de un fondo bibliográfico en temas afines a las Ciencias de la Salud.

\section{Resultados de un año de trabajo}

Para realizar un balance del trabajo ejecutado en el primer año de implantada la estrategia (año 2008), en los días comprendidos del 2 al 5 de febrero del 2009 se efectúo un taller en la Universidad de Ciencias Médicas de Holguín donde se valoró la situación actual del proceso de formación doctoral con énfasis en las actividades desarrolladas, situación de cada aspirante, logros e insuficiencias y propuesta de trabajo para este año. Los principales resultados obtenidos se exponen a continuación.

Durante los meses de enero y febrero del 2008 se dieron los pasos organizativos previos con la ENSAP para la formación de doctores en la Especialidad de Ciencias de la Salud, lo que se inició formalmente en el mes de marzo de ese año a partir del taller realizado en la Universidad de Ciencias Médicas de Holguín los días 24 y 25 , con la presencia de los profesores integrantes del departamento de Doctorados de la ENSAP y 17 profesionales de la salud del territorio, los que presentaron sus respectivos proyectos de investigación para su valoración como posibles temas de tesis doctorales.

Actualmente del grupo inicial de 17 quedan 13, pues tres de ellos se encuentran cumpliendo misión en Venezuela y una fue baja. De los 13, siete ya presentaron sus temas en el Consejo Científico Provincial, los que fueron aprobados y tres están 
listos para presentar. En estos momentos seis profesionales de los que iniciaron tienen sus temas aprobados por el CITMA.

Del grupo hay tres que tienen realizados los ejercicios de Idiomas y PSCyT. En los análisis efectuados por las diferentes partes que intervienen en la conducción de este proceso se valoró que hay tres profesionales rezagados respecto al resto, aun cuando el grupo en general podría mostrar resultados superiores.

Durante el año hubo nuevas inscripciones que incrementaron a 25 los profesionales incorporados al proceso de formación doctoral en Ciencias de la Salud en la provincia, en todos los casos se realizó una selección previa, mediante la presentación de sus trabajos en los talleres realizados a lo largo del año 2008.

Entre los profesionales hay ocho especialistas en Medicina General Integral, 14 en otras especialidades médicas, dos enfermeros y una psicóloga. De ellos 17 son mujeres y ocho son hombres. Seis se desempeñan en funciones de dirección a tiempo completo y 19 en actividades docentes, asistenciales e investigativas. Todos los integrantes del grupo tienen elaborado su plan de desarrollo individual, a partir del cual se hacen las valoraciones sobre el avance o no que van obteniendo en cada año, ya 18 tienen definidos sus tutores.

Durante el año se ejecutaron importantes actividades docentes, metodológicas y administrativas con los profesionales incorporados al proceso, entre ellas cabe mencionar:

- Cinco talleres con la participación en dúos de seis profesores de la ENSAP.

- Un curso sobre Problemas Sociales de la Ciencia y la Tecnología.

- Cinco conferencias especializadas impartidas por doctores en ciencias sobre diversos temas vinculados con Ciencias de la Salud.

- Entrevistas de asesorías a los profesionales por parte de profesores de la ENSAP, del Instituto "Pedro Kourí", del Instituto de Nutrición e Higiene de los Alimentos, el Instituto de Salud del Trabajador y de otras instituciones nacionales y locales.

- Creación de un fondo bibliográfico colectivo e individual a partir de la donación al Centro Provincial de Información de Ciencias Médicas por parte de la ENSAP, de ocho números de la Revista Cubana de Salud Pública y seis títulos de libros con temáticas de salud pública, los que fueron presentados por sus propios autores en cinco de ellos.

- Elaboración de un proyecto de investigación aceptado en el Programa Ramal de Investigaciones en Sistemas y Servicios de Salud.

- Elaboración y análisis de relatorías de cada taller y reuniones.

- Organización documental y realización de trámites establecidos.

- Organización interna del grupo con calendario quincenal de reuniones de carácter científico-metodológicas.

- Realización bimestral de una reunión con los directivos y el grupo para asegurar la marcha del proceso y su control. 
- Rendición de informes periódicos a las autoridades del territorio, de la ENSAP y Dirección Nacional de Postgrado del MINSAP.

- Apoyo sistemático a las necesidades del proceso por parte de la Dirección Provincial de Salud, la Universidad de Ciencias Médicas, la ENSAP, el metodólogo que dirige el proceso de doctorado y la directora de la Dirección Nacional de Postgrado del MINSAP.

En el balance efectuado también se valoraron algunas insuficiencias, tales como inasistencias e impuntualidad de algunos de los integrantes del grupo a las actividades programadas, así como en la entrega de documentación solicitada. Se apreció poco avance en la realización de publicaciones. Aún es reducido el número de aspirantes que han realizado exámenes de mínimos de Idiomas y PSCyT en relación con las potencialidades existentes.

Otro problema detectado es que la mayoría de los profesionales incorporados al proceso tienen serias carencias de tiempo para ejecutar la investigación a causa de sus responsabilidades laborales. Finalmente se consideró como un problema importante la insuficiencia de recursos propios para llevar a cabo con mayor efectividad las actividades de este proceso, entre ellos se destacan los financieros y la escasa disponibilidad de computadoras.

\section{Proyecciones de trabajo para el año 2009}

A partir del balance efectuado se trazaron las siguientes proyecciones para el año:

- Realización semestral de talleres de tesis.

- Definición de las necesidades individuales de asesorías y coordinación para la realización de las mismas.

- Adopción de medidas tendentes a incrementar la organización, control y disciplina de los participantes en el proceso por parte de los directivos de las instituciones de salud para lograr el cumplimiento de su cronograma y brindarles de forma efectiva las facilidades del tiempo y recursos necesarios.

- Consolidación de las tareas de organización de documentos y expedientes y su tramitación con los niveles correspondientes.

- Incremento de las publicaciones sobre los temas de investigación y ejecución de exámenes de mínimo, idiomas y PSCYT, o ambos para los que no lo han cumplido.

Para el sector de la salud en el territorio holguinero el proceso de formación doctoral en ciencias de la salud implementado constituye una oportunidad para incrementar el papel de la investigación científica en la solución de los problemas de salud y para los profesionales involucrados es un importante estímulo al ofrecerles una vía para su desarrollo científico, específicamente para alcanzar el grado de Doctor en Ciencias de la Salud desde sus escenarios laborales.

Los resultados que se aprecian en el primer año de trabajo de este proyecto ratifican la factibilidad de la participación local en la formación doctoral en Ciencias de la Salud y ponen de manifiesto una vez más, la importancia de la integración y de la intersectorialidad para la conducción de los procesos en el desarrollo de la salud pública. 


\section{REFERENCIAS BIBLIOGRÁFICAS}

1. Cuba. Gaceta Oficial. Ley No. 1281 de 2 de Diciembre de 1984.

2. Comisión Nacional de Grados Científicos (CNGC). Normas y Resoluciones vigentes para el desarrollo de los Grados científicos en la República de Cuba. La Habana: CNGC; 2005.

3. Grupo Asesor para el Doctorado del Ministerio de Salud Pública. Propuesta del Plan de Acción Estratégico para el desarrollo de los Grados Científicos del MINSAP. La Habana: MINSAP; 2006.

4. Programa de Doctorado Curricular en Ciencias de la Salud. La Habana:MINSAP; 2003.

5. Informe de la Delegación Territorial del CITMA. Holguín: Delegación; 2007.

6. Castañeda Abascal IE, Rodríguez Cabrera A, Hernández Meléndez E, Rojo Pérez $\mathrm{N}$. Universalización de la Formación académica e investigativa para la obtención del doctorado en el sector salud. Rev Cubana Salud pública [serie en Internet]. Dic 2008 [citado 10 Abr 2009 ];34(4): Disponible en:

http://scielo.sld.cu/scielo.php?script=sci arttext\&pid=S0864-

$\underline{34662008000400015 \& \operatorname{lng}=e s}$

Recibido: 7 de marzo de 2009.

Aprobado: 9 de mayo de 2009.

Alcides Abad Ochoa Alonso. Universidad de Ciencias Médicas de Holguín. Avenida Lenin No. 4 e/Arias y Agramonte. Holguín, Cuba.

E-mail: alcidesochoa@infomed.sld.cu 